$B J M G$ 10/2 (2007) 61-70

10.2478/v10034-008-0008-y

ORIGINAL ARTICLE

\title{
CHROMOSOMAL ABNORMALITIES IN ENDOMETRIAL AND OVARIAN CARCINOMAS
}

\author{
Pazarbaşi $\mathrm{A}^{1}{ }^{*}$, Kasap $\mathrm{M}^{1}$, Demirhan $\mathrm{O}^{1}$, Vardar $\mathrm{MA}^{2}$, \\ Suleymanova-Karahan $\mathrm{D}^{1}$, Doran $\mathrm{F}^{3}$
}

*Corresponding Author: Ayfer Pazarbaşi, Ph.D., Department of Medical Biology and Genetics, University of Çukurova, School of Medicine, 01330 Balcali, Adana, Turkey; Tel.: +90-322-338-70-68; Fax: +90-322-338-65-72; E-mail: payfer@cu.edu.tr

\begin{abstract}
Development and progression of human malignancies involve multiple genetic changes including chromosomal instabilities such as translocations, deletions, and inversions. Chromosomal abnormalities were observed in 23 cases with ovarian and endometrial cancer by cytogenetic studies using a GTG (G bands by trypsin using Giemsa) banding technique. Specific chromosome bands were frequently involved, and were most frequent on chromosomes 1 , 2, 3, 5, 12 and 17. Clonal alterations were observed at the cancer breakpoints, such as 1q21, 1q32, 3p21, $7 \mathrm{q} 22,11 \mathrm{q} 23$ in ovarian and $1 \mathrm{p} 36,1 \mathrm{q} 32,2 \mathrm{p} 12,3 \mathrm{p} 21$, 7q22, 9q34, 11p15, 11q23, 12q13, 14q11, 14q32, $16 \mathrm{p} 13,21 \mathrm{q} 22$ in endometrial cases. These findings provide evidence that multiple genetic lesions are associated with the pathogenesis of endometrial and ovarian cancer.
\end{abstract}

Key words: Endometrial cancer, Ovarian cancer, Cytogenetics

\footnotetext{
${ }^{1}$ Department of Medical Biology and Genetics, University of Çukurova, School of Medicine, Adana, Turkey

${ }^{2}$ Department of Obstetrics and Gynecology, University of Çukurova, School of Medicine, Adana, Turkey

${ }^{3}$ Department of Pathology, University of Çukurova, School of Medicine, Adana, Turkey
}

\section{INTRODUCTION}

Endometrial and ovarian carcinomas are the most common malignancies of the female genital tract. Most of these neoplasms are associated with chromosomal abnormalities and show a constellation of rearrangements such as translocations, deletions, and inversions [1-5]. However, understanding of the molecular genetic and cytogenetic changes associated with ovarian tumor development and disease progression has been limited because of the extreme complexity of the abnormalities [2]. Cytogenetic abnormalities have diagnostic and prognostic value in ovarian carcinoma $[2,5,6]$. Some chromosomal breakpoints and fragile sites observed in cancer cases are accepted as cancer breakpoints and fragile sites [7,8]. Among the 30 gene-richest bands, cancer breakpoints and fragile sites colocalize at 1p36, 1q21, $7 \mathrm{q} 22,8 \mathrm{q} 24,11 \mathrm{p} 15,11 \mathrm{q} 13,11 \mathrm{q} 23,12 \mathrm{q} 13,16 \mathrm{p} 13$, and 19 p13 $[7,8]$. Chromosome breakage is a manifestation of chromosomal instability. Chromosomal deletions in cancer cells are often found to contain tumor suppressor genes [7,8]. Structural rearrangements of chromosome arms 1p, 1q, 3p, 3q, 6q, 7p, 9q, 11p, $11 \mathrm{q}, 17 \mathrm{q}, 19 \mathrm{p}$, and $19 \mathrm{q}$ were identified in ovarian tumors $[1-3,5]$. Taken together, these findings suggest that certain chromosomal abnormalities are important in the pathogenesis of ovarian and endometrial cancers. Because of this, we carried out cytogenetic analyses after short-term cultures of 23 endometrial and ovarian tumors. We here describe the chromosomal changes we found. 


\section{MATERIALS AND METHODS}

Freshly operated tumor samples were obtained from 38 patients who had undergone surgery for endometrial and ovarian carcinomas at the hospital section of the Obstetrics and Gynocology at the Medical School of Çukurova University, Adana, Turkey. Fifteen were excluded from the study because of bacterial contamination, detachment, or presence of necrotic tissue, thus leaving 12 endometrial and 11 ovarian tumors for study. All tissues were examined macroscopically at the Pathology Department, Medical School, Çukurova University, Adana, Turkey, and came from invasive epithelial tumors. The study was approved by the Ethical Boards of the Medical School.

The tissues were disaggregated in a solution of trypsin-EDTA. The cell lines were initiated in tissue culture flasks $\left(25 \mathrm{~cm}^{2}\right)$ in Chang complete medium supplemented with penicillin-streptomycin to establish primary cultures [9]. The flasks were harvested for cytogenetic analysis after 7 to 10 days by exposure to colcemid followed by hypotonic treatment and fixation in methanol: acetic acid (3:1). The slides were incubated at $37^{\circ} \mathrm{C}$ for 2 days. Chromosomal analyses from tissue cultures were performed according to standart cytogenetic methods using the Giemsa banding technique [10]. The GTG (G bands by trypsin using Giemsa) banded slides were used for cytogenetic analysis and karyotype descriptions were made according to the International System for Human Cytogenetic Nomenclature (ISCN) [11].

\section{RESULTS}

Of the 38 tumor samples, 15 were excluded from the study because of bacterial contamination, detachment, or the presence of necrotic tissue. Karyotypic patterns were analyzed in 12 endometrial carcinomas and 11 ovarian carcinomas. All samples were from invasive epithelial tumors and characterized histologically as follows: of the endometrial tumors, four were at grade III, seven at grade II and one at grade I, while of the ovarian tumors, one was at grade III, five at grade II and five at grade I. The patients did not all show the same stage of cancer progression and there was no relationship between the complexity of chromosome constitution and the cancer progression.

Structural alterations present in more than one cell were termed clonal alterations. Thirteen patients had clonal chromosomal abnormalities, eight had non clonal abnormalities, and two cases had normal karyotypes. Most specimens exhibited complex karyotypes with many numerical and structural changes (Table 1). Numerical changes were seen in six cases: case O5 had karyotype 54,XX with numerical changes at chromosomes 9, 10,12,14, 15 and 20. Aneuploidy was observed in case E5. Cases O11, E1, E6 and E7 showed near-triploid, tetraploid and polyploid modal numbers with heterogenous structural abnormalities. Eleven showed a variety of structural abnormalities and five had only one structural abnormality. Deletions, translocations, inversions, gaps or breaks, acentric fragments, double minutes, radials and endoreduplications were observed in varying proportions. Translocations were found in four cases. Case E1 had $\mathrm{t}(19 ; 22)(\mathrm{q} 13 ; \mathrm{p} 13)$ and $\mathrm{t}(21 ; 22)(\mathrm{p} 13 ; \mathrm{q} 13)$, while case E2 had $\mathrm{t}(14 ; 21)(\mathrm{q} 11 ; \mathrm{q} 11)$, case O5 had $\mathrm{t}(3 ; 7)(\mathrm{q} ; \mathrm{q})$ and case $\mathrm{O} 4$ had $\mathrm{t}(1 ; 22)$ (qter;p). Two cases (13 cells of E6 and five cells of O11) had double minutes and one case with triradial and quadriradials (seven cells of E6) (Table 1).

The cancer breakpoints and fragile sites observed are shown in Table 2. Chromosomes 3, 5, 1, 12, 17 and 2 (cited according to their frequency) were most frequently involved in structural abnormalities (Table 3 and 4 and Figure 1). Structural rearrangements acquired on chromosomes 2, 4, 7, 8, 9, 10, 11, 13, 15, 17,18 and $\mathrm{X}$ in ovarian cancers and on chromosomes $14,19,20$ and 22 in endometrial cancers.

\section{DISCUSSION}

Understanding the molecular basis of the initiation and progression of ovarian and endometrial cancer will be strongly dependent on advanced cytogenetic and molecular genetics. In this cytogenetic study we described the chromosomal findings detected in 23 endometrial and ovarian tumors, only two had normal karyotypes, and possibly fibroblast-derived instead of tumorigenic tissues [12-14]. Although five tumors revealed only one simple structural change, the karyotypes of the other 16 tumors were quite complex. Our results indicate that several chromosomal regions were non randomly involved in abnormalities. The patients did not all show the same stage of histological grade and there was no relationship between the complexity of chromosome constitution 
Table 1. Chromosomal abnormalities in 12 endometrial (E1-12) and 11 ovarian (O1-11) carcinomas. The complete karyotypes are listed. When more than one karyotype was identified within individual samples, the number of cells of each type is shown in square brackets.

\begin{tabular}{|c|c|c|c|}
\hline Case \# & No of $M^{*}$ & $\mathbf{H G}^{* *}$ & Endometrial Cancer \\
\hline E1 & 35 & III & $\begin{array}{l}\text { 40 79,XX, fra(1q11) (q32), fra(3p21), fra(5q31), fra(7q22), fra(9q34), fra(11q23), fra(14q24), } \\
\text { fra(15q22), fra(17q21), del(12p13), dup(2p11-12), inv11(p15;q23), inv(21p13;q22)[2], } \\
t(19: 22)(q 13 ; p 13), t(21 ; 22)(p 13 ; q 13), 2 p+[2]\end{array}$ \\
\hline E2 & 26 & II & $\begin{array}{l}\text { 46;XX, chtb(2p13), chtb(5q31)[3], chtb(7q22), chtb(17q21)[7], del(7q34), del(11p15), } \\
\text { fra(11q23), fra(Xq27), i(2p13), t(14;21)(q11;q11) }\end{array}$ \\
\hline E3 & 22 & II & $46 ; X X, \operatorname{chtb}(5 q 31), \operatorname{del}(11 \mathrm{p} 15),(q 23)$, fra(17q21) \\
\hline E4 & 57 & I & $46 ; X X, \operatorname{gap}(1 \mathrm{p} 36),(\mathrm{q} 32)[2], \operatorname{gap}(3 \mathrm{p} 21), \operatorname{gap}(5 \mathrm{q} 31)[4]$, fra $(5 \mathrm{q} 31)[2]$, fra(7q22), fra(14q32) \\
\hline E5 & 41 & III & $\begin{array}{l}\text { 25-46,XX, gap(1p21),(p36),(q32), gap(3p14)[4],(q21)[2], gap(5q22),(q31), chtb(1p13), } \\
\operatorname{del}(3 \mathrm{q} 21, \mathrm{p} 21), \operatorname{del}(5 \mathrm{p} 15), \operatorname{fra}(5 \mathrm{q} 31)[2], \operatorname{fra}(6 \mathrm{q} 25), \operatorname{fra}(11 \mathrm{q} 23), \operatorname{inv}(9)(\mathrm{q} 12 ; 34), \text { heteroploidi(7) } \\
(\mathrm{q} 22), 1 \mathrm{ph}+, 3 \mathrm{qh}+\end{array}$ \\
\hline E6 & 85 & II & $\begin{array}{l}\text { 19-141,XX, del(1p13),(p21)[4],(p36)[2],(q32)[3], del(3p21,q21)[8],(q25)[2],(p14,q21), } \\
\text { del(4p15)[2], del(5p15), del(6q11),(q15),(q21), del(9q12), del(12p13)[4], del(16q23), } \\
\operatorname{del}(X \mathrm{X} 26)[2], \operatorname{fra}(1 \mathrm{p} 21),(\mathrm{p} 36),(\mathrm{q} 42), \operatorname{fra}(4 \mathrm{q} 21), \operatorname{fra}(5 \mathrm{q} 31)[3], \text { fra(10p13)[2], fra(12q13), } \\
\text { fra(13q22), fra(14q24), fra(16p13), fra(X)(p22),(q27), chtb(1p36)[2],(q21)[2], } \\
\operatorname{chtb}(2 \mathrm{p} 13),(\mathrm{q} 11)[2],(\mathrm{q} 21)[2],(\mathrm{q} 35)[3], \operatorname{chtb}(3 \mathrm{p} 21)[2], \operatorname{chtb}(4 \mathrm{p} 11),(\mathrm{p} 15),(\mathrm{q} 11),(\mathrm{q} 21), \\
\operatorname{chtb}(5 \mathrm{q} 12),(\mathrm{q} 31), \operatorname{chtb}(6 \mathrm{q} 21), \operatorname{chtb}(7 \mathrm{q} 11), \operatorname{chtb}(9 \mathrm{q} 22)[3], \operatorname{chtb}(10 \mathrm{p} 13), \operatorname{chtb}(12 \mathrm{p} 13),(\mathrm{q} 13)[2], \\
\text { chtb(16p13), chtb(17p13)[2],(q11),(q21), chtb(20p11), chtb(Xq26), gap(12q24), gap(13q14) } \\
\text { [3],(q22)[3], gap(15q15)[3], dup(5q22), der(7,17)(p,p),i(Xq),dm[13], ace, tr and qr [7] }\end{array}$ \\
\hline E7 & 79 & III & $\begin{array}{l}\text { 38-92,XX, gap(1q32), gap(3p21)[3], fra(1)(q21),(q32)[4], fra(3p21), fra(5q31)[8], fra(9q34), } \\
\text { fra(10q23), fra(12q13), fra(17q25), del(4p15), del(5p15)[3], del(21q22), 13p+ }\end{array}$ \\
\hline E8 & 12 & II & $46 ; X X, f r a(3 p 14)$, fra(6q21), fra(12q24)[2], fra(Xq26) \\
\hline E9 & 60 & II & $46 ; X X, \operatorname{del}(3 p 25), \operatorname{chtb}(3 q 11)$, fra(9q34), fra(11q23) \\
\hline E10 & 18 & II & $46 ; X X$, fra(12q24) \\
\hline E11 & 11 & II & $46 ; X X$, fra $(2 q 35)$ \\
\hline E12 & 42 & III & $46 ; X X$ \\
\hline Case \# & No of $M *$ & $\mathbf{H G}^{* *}$ & Ovarian Cancer \\
\hline $\mathrm{O} 1$ & 60 & II & $\begin{array}{l}\text { 46,XX, fra(1)(q21), (q32), fra(2q35), fra(3)(p25),(q27), fra(7q22), fra(1q23), fra(14) } \\
(\mathrm{q} 11),(\mathrm{q} 24), \operatorname{chtb}(1 \mathrm{p} 36), \operatorname{chtb}(5 \mathrm{q} 12), \operatorname{chtb}(10 \mathrm{q} 23), \operatorname{chtb}(12 \mathrm{q} 13), \operatorname{chtb}(14 \mathrm{q} 11), \operatorname{del}(2 \mathrm{q} 35), \\
\text { del(18q23) }\end{array}$ \\
\hline $\mathrm{O} 2$ & 20 & II & $46, X X, \operatorname{fra}(1 \mathrm{q} 21), \operatorname{chtb}(4 \mathrm{q} 11), \operatorname{chtb}(5 \mathrm{q} 31), \operatorname{chtb}(17 \mathrm{q} 24)$ \\
\hline $\mathrm{O} 3$ & 35 & I & $46, X X$, fra(1q32), chtb(8q21) \\
\hline $\mathrm{O} 4$ & 10 & III & 46,XX, chtb(1q21), t(22p;1qter) \\
\hline O5 & 35 & II & $54, \mathrm{XX},+9 \times 3,+10 \times 3,+12 \times 5,+14,+15,+20 \times 3[35] / \mathrm{t}(3 \mathrm{q} ; 7 \mathrm{q})$ \\
\hline O6 & 42 & I & $46, X X, \operatorname{gap}(5 q 31)[2]$ \\
\hline $\mathrm{O} 7$ & 24 & I & $46, \mathrm{XX}, 2 \mathrm{qh}+, \operatorname{fra}(3)(\mathrm{p} 14)[2],(\mathrm{p} 21),(\mathrm{q} 11)[2],(\mathrm{q} 21),(\mathrm{q} 27), 9 \mathrm{qh}+$ \\
\hline O8 & 19 & II & 46,XX, gap(1q21) \\
\hline O9 & 11 & I & $46, \mathrm{XX}$ \\
\hline $\mathrm{O} 10$ & 48 & II & $46, \mathrm{XX}, 2 \mathrm{qh}+[13]$ \\
\hline O11 & 27 & I & $\begin{array}{l}\text { 47-77,XX, gap(2q23), chtb(3)(p25)[2],(q11), } \operatorname{chtb}(9 \mathrm{p} 22), \operatorname{dup}(4 \mathrm{q} 11 \rightarrow \operatorname{ter})[27] \\
\operatorname{dup}(18 \mathrm{q} 11 \rightarrow \operatorname{ter})[27], \operatorname{del}(4 \mathrm{p} 15), \operatorname{del}(5 \mathrm{p} 15)[5], \operatorname{del}(11 \mathrm{q} 23), \operatorname{del}(13 \mathrm{p} 11)[2], \operatorname{del}(15 \mathrm{p} 11), \\
\operatorname{inv}(12 \mathrm{p} 10 ; \mathrm{q} 13)[3], \operatorname{dm}[5] /+12[7]\end{array}$ \\
\hline
\end{tabular}

*Metaphases; **Histological Grade 
Table 2. Breakpoints and clonal alterations observed per chromosome in endometrial and ovarian cancer. Boldface represents cancer breakpoints.

\begin{tabular}{|c|c|c|}
\hline $\begin{array}{c}\text { Chromosome } \\
\text { Number }\end{array}$ & Endometrial Cancer & Ovarian Cancer \\
\hline 1 & $1 \mathrm{p} 13[2], \mathrm{p} 21[6], \mathbf{p 3 6}[7], \mathrm{q} 11, \mathbf{q} 21[3], \mathbf{q} 32[12], \mathrm{q} 42$ & 1p36, q21[4], q32[2] \\
\hline 2 & 2p13[2], q11[2], q21[2], q35[4] & $2 \mathrm{q} 23, \mathrm{q} 35[2]$ \\
\hline 3 & p14[6], p21[17], p25, q11, q21[11], q25[2] & $\mathrm{p} 14[2], \mathbf{p} 21, \mathrm{p} 25[3], \mathrm{q} 11[3], \mathrm{q} 21, \mathrm{q} 27[2]$ \\
\hline 4 & $4 \mathrm{p} 11, \mathrm{p} 15[3], \mathrm{p} 16, \mathrm{q} 11, \mathrm{q} 21[2]$ & $4 \mathrm{p} 15, \mathrm{q} 11[2]$ \\
\hline 5 & $5 \mathrm{p} 15[5], \mathrm{q} 12, \mathrm{q} 22[2], \mathrm{q} 31[27]$ & $5 \mathrm{p} 15[5], \mathrm{q} 12, \mathrm{q} 31[3]$ \\
\hline 6 & $6 \mathrm{q} 11, \mathrm{q} 15, \mathrm{q} 21[3], \mathrm{q} 25$ & \\
\hline 7 & 7q11, q22[4], q34 & $7 q 22$ \\
\hline 8 & & $8 \mathrm{q} 21$ \\
\hline 9 & $9 q 12, q 22[3], \mathbf{q 3 4}[3]$ & $9 \mathrm{p} 22$ \\
\hline 10 & $10 \mathrm{p} 13[3], \mathrm{q} 23$ & $10 \mathrm{q} 23$ \\
\hline 11 & $11 \mathrm{p} 15[3], \mathbf{q 2 3}[7]$ & $11 q 23[2]$ \\
\hline 12 & $12 p 13[6], \mathbf{q 1 3}[4], q 24[4]$ & $12 q 13$ \\
\hline 13 & $13 q 14[3], \mathrm{q} 22[4]$ & $13 p 11$ \\
\hline 14 & 14q11, q24[2], q32 & $14 q 11[2], q 24$ \\
\hline 15 & $15 \mathrm{q} 15, \mathrm{q} 22$ & $15 \mathrm{p} 11$ \\
\hline 16 & $16 p 13[2], q 23$ & \\
\hline 17 & $17 \mathrm{p} 13[2], \mathrm{q} 11, \mathrm{q} 21[10], \mathrm{q} 25$ & $17 \mathrm{q} 24$ \\
\hline 18 & & $18 \mathrm{q} 11, \mathrm{q} 23$ \\
\hline 20 & $20 \mathrm{p} 11$ & \\
\hline 21 & $21 q 22$ & \\
\hline $\mathrm{X}$ & $\mathrm{Xp} 22, \mathrm{q} 26[3], \mathrm{q} 27[2]$ & \\
\hline
\end{tabular}

and cancer progression.

Chromosomes 3, 5 and 1 were (sequenced according to their frequency) most frequently involved in structural abnormalities in both endometrial and ovarian cases. Involved in structural clonal alterations were chromosomes $1,2,3,4,5,6,7,9,10$, $11,12,13,16,17,21, \mathrm{X}$ in endometrial cases, and chromosomes $1,3,5,12,14$ in ovarian cases. In endometrial cases, deletion of chromosome 3 , the most frequent structural alteration observed, was found in 13 cells of cases E5, E6 and E9, while no deletion of chromosome 3 was found in ovarian cancers. Deletions generally cause the loss of tumor suppressor genes [15-19]. Molecular studies have shown loss of heterozygosity ( $\mathrm{LOH})$ on $3 p$, which seems to be a common event in endometrial tumors and related to LOH in the FHIT (fragile histidine triad) and the $h M L H 1$ locus [13]. According to Imamura et al. [13], these losses contribute to the progression of the tumor to an undifferentiated stage, aggressive behavior, or metastatic potential. In another study [14], various deletions $(3 p 13,21,22,26)$ of chromosome $3 p$ were associated with the development of uterine cervical carcinoma in Indian patients. Del(3)(q13q23) was the most consistent aberration observed in six of nine gastric and esophageal adenocarcinomas [15]. Through positional cloning of the $3 \mathrm{q} 21$ breakpoint, a novel gene, $D I R C 2$, reported to encode a multi membrane-spanning protein that represents a new member of the major facilitator superfamily (MFS) of transporters, and these fragilities associated with sporadic and familial renal cell carcinomas [16].

Although the second most frequent clonal alterations that we observed was located on chromosome 5 , the most frequent clonal alteration we observed in both tumor groups was $5 \mathrm{q} 31$. The q31 band of chro- 

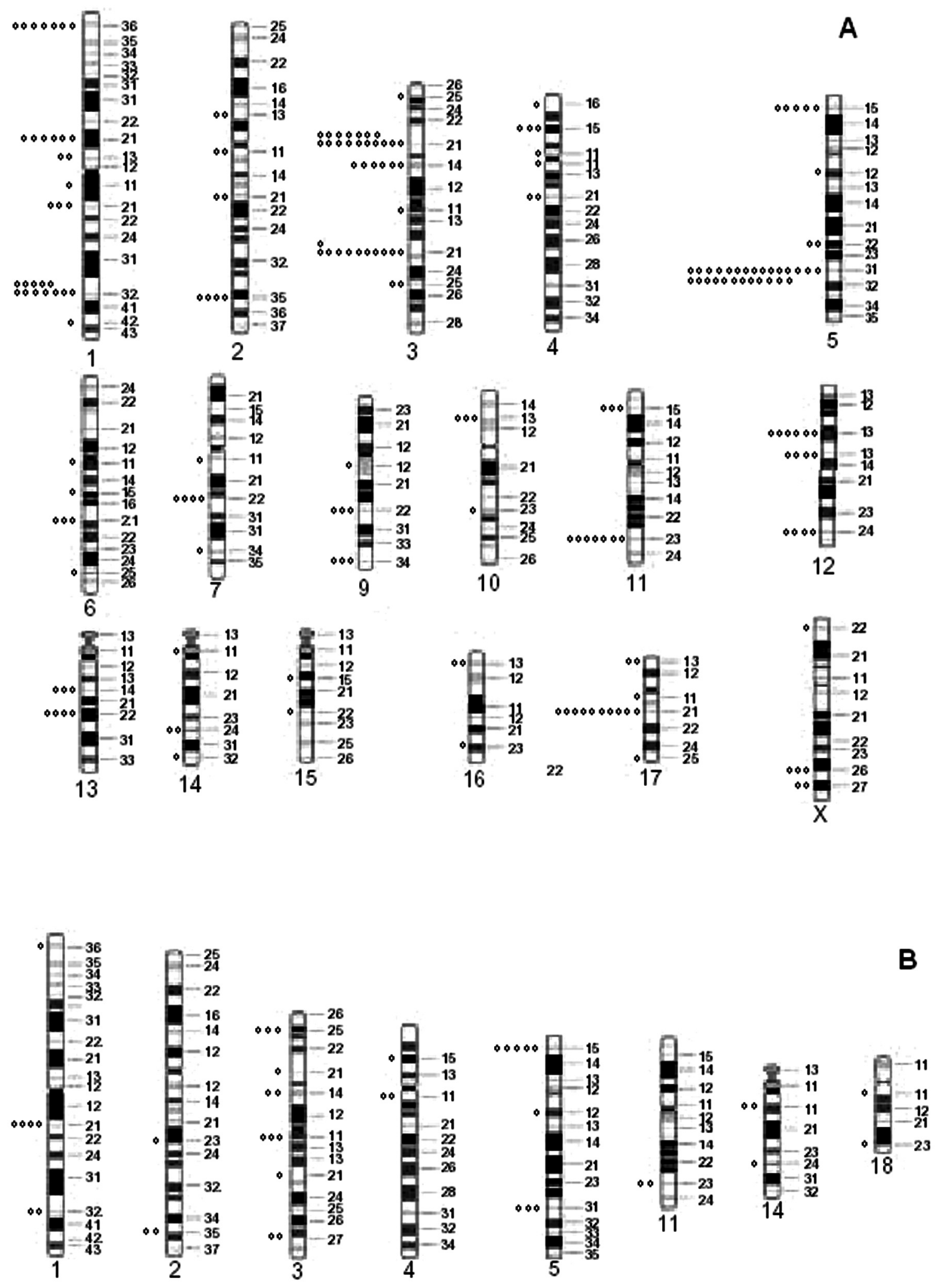

Figure 1. Localization of breakpoints and clonal alterations observed in endometrial (A) and ovarian (B) cancers. 
Table 3. Frequency of abnormalities observed per chromosome in endometrial cancer (listed in decreasing order).

\begin{tabular}{|c|c|c|}
\hline $\begin{array}{c}\text { Chromosome } \\
\text { Number }\end{array}$ & $\begin{array}{c}\text { Number of Abnormalities } \\
\text { (total metaphases analyzed) }\end{array}$ & Abnormality (\%) \\
\hline 3 & $40(488)$ & $\mathbf{8 . 2 0}$ \\
\hline 5 & $35(488)$ & $\mathbf{7 . 1 7}$ \\
\hline 1 & $32(488)$ & $\mathbf{6 . 5 5}$ \\
\hline 12,17 & $14(488)$ & $\mathbf{2 . 8 6}$ \\
\hline 2 & $12(488)$ & $\mathbf{2 . 4 5}$ \\
\hline 9,11 & $9(488)$ & $\mathbf{1 . 8 4}$ \\
\hline 4 & $8(488)$ & $\mathbf{1 . 6 3}$ \\
\hline 13 & $7(488)$ & $\mathbf{1 . 4 3}$ \\
\hline $6,7, X$ & $6(488)$ & $\mathbf{1 . 2 2}$ \\
\hline $10,14,21$ & $4(488)$ & 0.81 \\
\hline 16 & $3(488)$ & 0.61 \\
\hline $15,18,22$ & $2(488)$ & 0.40 \\
\hline 19,20 & $1(488)$ & 0.20 \\
\hline
\end{tabular}

Table 4. Frequency of abnormalities observed per chromosome in ovarian cancer (listed in decreasing order).

\begin{tabular}{|c|c|c|}
\hline Chromosome Number & $\begin{array}{c}\text { Number of Abnormalities } \\
\text { (total metaphases analyzed) }\end{array}$ & Abnormality (\%) \\
\hline 3 & $12(315)$ & $\mathbf{3 . 8 0}$ \\
\hline 5 & $9(315)$ & $\mathbf{2 . 8 5}$ \\
\hline 1,12 & $7(315)$ & $\mathbf{2 . 2 2}$ \\
\hline $2,4,14$ & $3(315)$ & 0.95 \\
\hline 11,18 & $2(315)$ & 0.63 \\
\hline $7,8,9,10,13,15,17$ & $1(315)$ & 0.31 \\
\hline
\end{tabular}

mosome 5 has been the subject of intensive studies as it is a gene-rich area. It contains genes of known biological function, including growth factor, growth factor receptor, hormone receptor and neurotransmitter receptor [17].

The third most frequent clonal alteration that we observed was located on chromosome 1 . One of the clonal alteration in endometrial cases was at $1 \mathrm{p} 36$. Four genes, mapping to $1 \mathrm{p} 36$, have been proposed as candidate tumor suppressor genes: Heir-1 (ID3), a neuroectodermally expressed member of the Id family of developmental negative regulatory genes; $T N F R 2$, one of two tumor necrosis factor receptor genes; PITSLRE, a cell cycle-regulated kinase gene with homology to human $C D C 2$; and $D A N$, a transcription factor gene homologous to a mouse tumor suppressor gene. Two additional genes, the transcription factor regulator $E 2 F 2$ and the paired-box-containing gene $P A X 7$, were recently mapped to $1 \mathrm{p} 36$ and are thus additional potential candidate suppressor genes. Disruption of one or more of these candidate genes could play a role in the development of endometrial cancer [6].

The 12q13-15 region was involved in six cells of three cases in our study. It contains several genes (including an oncogene) of potential importance for 
neoplastic development, for example, $S A S, S P 1$, INT-1, GLI, CHOP, CDK4 and MDM2 [19-21]. Molecular studies have shown that these genes are often coamplified, although there have also been cases with only one or two of these genes included in the amplicon [20,21]. The presence of an additional copy of chromosome 12 with an altered gene at 12q13 may contribute to the development of a malignancy. The $H M G I-C$ gene encodes a member of the high-mobility-group proteins, which are non histone nuclear proteins able to bind AT-rich regions in the minor groove of DNA through three so-called AT-hook domains and may affect transcription by acting as architectural proteins. They have been found overexpressed in human and experimental malignancies [21]. Rearrangements of the $H M G I-C$ gene are the consequence of chromosomal aberrations involving the 12q13-15 region where the $H M G I-C$ gene is located [21].

Based on data on breakpoints at the 2q35-q37 region, a genetic link between some carcinomas has been suggested for a common pathway of the development of these tumors. This region include paired box protein (PAX3) gene [22]. However, the role of the PAX3 gene in rearrangements in chromosome 2 in endometrial and ovarian carcinomas remains to be determined.

The THY1 gene was mapped to the 11q23 24, which has been identified by both Taetle et al. [1] and Garba et al. [22] to have a high incidence of LOH in ovarian cancer. The location of the THY1 gene at this region makes this a suitable tumor suppressor gene for ovarian cancer. The THYl gene was found to be exclusively expressed in the two non tumorigenic cell clones [23]. In contrast, THY1 expression was not determined in the tumorigenic ovarian cell lines. We observed clonal alterations at this region in both carcinoma types in our study. The mechanism of how THY1 expression is abolished in ovarian cancer cells remains elusive.

We observed fragility at 7q22 in both endometrial and ovarian tumors. That observed in endometrial tumors was a clonal alteration. Genes mapped to the 7q22 region include the human cut-like 1 gene (CUTL1), human mismatch repair gene (PMS2L), erythropoietin gene $(E P O)$, and asparagine synthetase gene (ASNS). The fact that most structural rearrangements involving 7q22, suggest the hypothesis that this site contains a critical region of single or multiple genes, that when mutated or deleted results in malignant transformation. Whether this involves a structural alteration of a putative tumor suppressor gene remains to be clarified [24].

Two main cytogenetic subgroups have been identified in published cases of ovarian tumors with chromosome abnormalities: tumors with simple karyotypic changes and tumors with complex karyotypic abnormalities. Simple karyotypic changes are defined as simple numerical changes, whereas complex karyotypic changes are multiple numerical and structural abnormalities of chromosomes. We found that aberrations may be of significance in the pathogenesis of ovarian cancer and may provide valuable information for further investigation at the molecular level. Simple karyotypic changes, including trisomy $12,10,7,8,5$ (in decreasing order of frequency) have been considered as primary chromosomal changes in ovarian cancer [5]. The 54,XX karyotype with complex numerical changes at chromosomes 9, 10, $12,14,15$ and 20, which we found in case O5, have not been previously reported. These chromosomes may play a specific role in the pathogenesis of ovarian cancer. Because trisomy 10 has been observed in some benign tumors and in normal tissues that surround malignant tumors, it may contribute to the transformation of normal tissues and/or the initiation of tumorigenesis [5]. Marked aneuploidy represents this aggressive phenotypic behavior at a cytogenetic level. However, chromosomal aberrations need not necessarily be associated with advanced-stage disease. We observed two normal 46,XX karyotypes in a highly malignant endometrial (E12) and an ovarian (O9) cancer.

In conclusion, this study has shown that specific chromosome bands, frequently involved in clonal alterations and breakpoints, may indicate the regions in which to search for new dominant oncogenes or recessive tumor suppressor genes. Our cytogenetic findings provide strong evidence that multiple genetic lesions are associated with the development of endometrial cancers, and that deletions of $3 \mathrm{p} 13$, p21, p23, q21 and q25 may play a specific role in the pathogenesis of such cancers. Chromosomal abnormalities have been known to occur in malignant tumors for a long time [1-5]. This study adds little to the understanding of the field of ovarian cancer cytogenetics; the results for endometrial cancer are more novel and could be improved by analyses of more cases. 


\section{ACKNOWLEDGMENTS}

This study was supported by the Department of Research Projects at the University of Çukurova, Adana, Turkey.

\section{REFERENCES}

1. Taetle R, Aickin M, Yang LM, Panda L, Emerson J, Roe D, Adair L, Thompson F, Liu Y, Wisner L, Davis JR, Trent J, Alberts DS. Chromosome abnormalities in ovarian adenocarcinoma: I. Nonrandom chromosome abnormalities from 244 cases. Genes Chromosomes Cancer 1999; 25(3): 290-300.

2. Deger RB, Faruqi ShA, Noumoff JS. Karyotypic analysis of 32 malignant epithelial ovarian tumors. Cancer Genet Cytogenet 1997; 96(2): 166-173.

3. Thompson FH, Taetle R, Trent JM, Liu Y, Massey-Brown K, Scott KM, Weinstein RS, Emerson JC, Alberts DS, Nelson MA. Band 1p36 abnormalities and $\mathrm{t}(1 ; 17)$ in ovarian carcinoma. Cancer Genet Cytogenet 1997; 96(2): 106-110.

4. Couturier J. Uterus: endometrial carcinoma. Atlas Genet Cytogenet Oncol Haematol (Elecronic journal) 1999 (http://www.infobiogen.fr/services/chromcancer/Tumors/endometrID5045.html)

5. Wang JCh, Masson AMM, Tonin PN, Provencher, Eydoux P. Trisomy of chromosome 10 in two cases of ovarian carcinoma. Cancer Genet Cytogenet 2000; 118(1): 65-68.

6. Thompson FH. Cytogenetic methods and findings in human solid tumors. In: Barch MJ, Knutsen T, Spurbeck J, eds. The ACT Cytogenetics Laboratory Manual, 3rd ed. Philadelphia: Lippincott Raven. 1997; 98-118.

7. Musio A, Mariani T, Vezzoni P, Frattini A. Heterogeneous gene distribution reflects human genome complexity as detected at the cytogenetic level. Cancer Genet Cytogenet 2002; 134(2): 168-171.

8. Musio A, Zambroni D, Vessoni P, Mariani T. Chromosomes, genes and cancer breakpoints. Cancer Genet Cytogenet 2002; 139(2): 141-142.

9. Mandahl N. Methods in solid tumour cytogenetics. In: Rooney DE, Czepulkowski BH, eds. Human Cytogenetics: A Practical Approach, 2nd ed. Oxford: IRL Press at Oxford University Press. 1992; 155-187.

10. Köhler A. Chromosome staining. In: Wegner RD, ed. Diagnostic Cytogenetics. Berlin: SpringerVerlag. 1999; 56-60.
11. ISCN 1995: an international system for human cytogenetic nomenclature (1995): recommendations of the International Standing Committee for Human Cytogenetic Nomenclature, Memphis, TN, USA, October 9-13, 1994. In: Mitelman F, ed. Basel: Karger. 1995.

12. 13. Imamura $T$, Arima $T$, Kato $H$, Miyamoto $S$, Sasazuki T, Wake N. Chromosomal deletions and Kras gene mutations in human endometrial carcinomas. Int J Cancer 1992; 51(1): 47-52.

13. Argüden YT, Hacihanefioğlu S. Kanser sitogenetiği. Klinik Gelisim 2000; 13(8-9-10): 330-335 (in Turkish).

14. Dasgupta S, Chakraborty SB, Roy A, Roychowdhury S, Panda CK. Differential deletions of chromosome $3 p$ are associated with the development of uterine cervical carcinoma in Indian patients. J Clin Pathol Mol Pathol 2003; 56(5): 263-269.

15. Rao PH, Mathew S, Kelsen DP, Chaganti RSK. Cytogenetics of gastric and esophageal adenocarcinomas. Cancer Genet Cytogenet 1995; 81(2): 139-143.

16. Bodmer D, Janssen I, Jonkers Y, Berg E, Dijkhuizen T, Debiec-Rychter M, Schoenmakers E, Kessel AG. Molecular cytogenetic analysis of clustered sporadic and familial renal cell carcinoma-associated 3q13 q22 breakpoints. Cancer Genet Cytogenet 2002; 136(2): 95-100.

17. Guenet L, Henry C, Toutain B, Dubourg C, Le Gall JY, David V, Le Treut A. Eucaryotic translation termination factor gene (ETF1/eRF1) maps at D5S500 in a commonly deleted region of chromosome $5 \mathrm{q} 31$ in malignant myeloid diseases. Cytogenet Cell Genet 2000; 88(1-2): 82-86.

18. Gisselsson D, Andreasson P, Meis-Kindblom JM, Kindblom LG, Mertens F, Mandahl N. Amplification of $12 \mathrm{q} 13$ and $12 \mathrm{q} 15$ sequences in a sclerosing epitheloid fibrosarcoma. Cancer Genet Cytogenet 1998; 107(2): 102-106.

19. Stock AD, Dennis TR. A translocation breakpoint at chromosome band 12q13 associated with B-cell chronic lymphocytic leukemia. Cancer Genet Cytogenet 1999; 111(2): 166-168.

20. Santulli B, Kazmierczak B, Napolitano R, Caliendo I, Chiappetta G, Rippe V, Bullerdiek J, Fusco A. A 12 q13 translocation involving the HMGI-C gene in richter transformation of a chronic lymphocytic leukemia. Cancer Genet Cytogenet 2000; 119(1): 70-73.

21. Yeh YA, Rao PH, Cigna CT; Middlesworth W, Lefkowitch JH, Murty VVVS. Trisomy 1q, 2 and 20 
in a case of hepatoblastoma: possible significance of 2q35-q37 and 1q12-q21 rearrangements. Cancer Genet Cytogenet 2000; 123(2): 140-143.

22. Gabra H, Watson JEV, Taylor KJ, Mackay J, Leonard RCF, Steel CM, Porteous DJ, Smyth JF. Definition and refinement of a region of loss of heterozygosity at 11q23.3-q24.3 in epithelial ovarian cancer associated with poor prognosis. Cancer Res 1996; 56(5): 950-954.
23. Abeysinghe HR, Cao Q, Xu J, Pollock S, Veyberman Y, Guckert NL, Keng P, Wang N. THY1 expression is associated with tumor suppression of human ovarian cancer. Cancer Genet Cytogenet 2003; 143(2): 125-132.

24. Forrest DL, Lee CL. Constitutional rearrangements of 7q22 in hematologic malignancies: a new case report. Cancer Genet Cytogenet 2002; 139(1): 75-77. 
